\title{
Alcohol Consumption in Patients with Psoriasis and its Relationship to Disease Severity
}

\author{
Ermira Vasili ${ }^{1, *}$, Sidita Borici ${ }^{1}$, Erisa Alia ${ }^{1}$, Elna Cano $^{1}$, Loreta Kuneshka $^{2}$ and Iris Hoxha ${ }^{3}$ \\ ${ }^{1}$ Dermatology Department, Mother Teresa University Hospital, Rruga e Dibres, 372, Tirana, Albania \\ ${ }^{2}$ Statistics Department, University of Medicine, Rruga e Dibres, 371, Tirana, Albania \\ ${ }^{3}$ Pharmacy Department, Mother Teresa University Hospital, Rruga e Dibres, 372, Tirana, Albania
}

\begin{abstract}
Moderate to severe psoriasis is associated with increased alcohol intake and excessive mortality from alcoholrelated causes. Alcohol biomarkers are those providing an objective measurement of its consumption. The objective of this study is to assess alcohol consumption in a cohort of patients with psoriasis and to investigate the influence of alcohol intake on disease severity. Patients with psoriasis hospitalized at the Clinic of Dermatology, in Mother Teresa University Hospital were recruited in this cross- sectional study. Alcohol consumption was assessed via CAGE questionnaire and the self- reported amount of alcohol consumed, whereas disease severity was evaluated via PASI scoring system. Blood specimens were taken at admission and levels of gamma glutamyltransferase (GGT), alanine aminotransferase (ALT), aspartate aminotransferase (AST) and mean corpuscular volume of erythrocytes (MCV) were measured. Statistical analysis was performed using SPSS 20.0 statistical package. A total of 62 in- patients completed the study. Significant correlations were observed between GGT and AST values with raki and beer consumption, ALT value with raki, beer and wine consumption and MCV value with raki consumption. Disease severity did not correlate significantly with raki and beer consumption ( $p>0.05$ ). Logistic regression analysis between Psoriasis Area and Severity Index (PASI) score and raki consumption in male patients with psoriasis duration of more than 3 years resulted in statistical significance $(b=23.5, p<0.05)$. Combination of parameters related to chronic alcohol consumption offers advantage over every isolated test. Measurement of simple laboratory parameters combined with self- report methods of consumption allows identification of users.
\end{abstract}

Keywords: Alcohol consumption, psoriasis severity, Albania, alcohol biomarkers, raki consumption.

\section{INTRODUCTION}

Moderate to severe psoriasis is associated with increased alcohol intake and excessive mortality from alcohol-related causes [1]. Although the methods of self- report offer a reliable and valid approach to measuring alcohol consumption [2], alcohol biomarkers are those providing an objective measurement of its consumption [1]. The effectiveness of screening of single biomarkers has been shown to be insufficient [3], but combination of parameters linked to chronic drinking by different pathophysiological mechanisms, e.g. carbohydrate- deficient transferrin (CDT), gamma glutamyltransferase (GGT) and mean corpuscular volume of erythrocytes (MCV) offers better benefit in detection of alcohol consumption over any isolated test. The serum level of GGT, aspartate aminotransferase (AST) and alanine aminotransferase (ALT) is a sum of drinking activity and liver status; MCV on the other hand seems to be raised with regular drinking, although in alcoholics it may continue to raise despite drinking cessation since the life-span of erythrocytes is 120 days. Alcohol misuse for long has been suspected to

*Address correspondence to this author at the Dermatology Department, Mother Teresa University Hospital, Rruga e Dibres, 372, Tirana, Albania; Tel: 00355 692058153; Fax: 00355 42362627; E-mail: miravasili@hotmail.com contribute to psoriasis pathology, despite numerous difficulties in verification of a relation between its consumption and the disease presentation [4]. Many studies have found a relationship between alcohol consumption and psoriasis as far as it concerns poorer treatment response or gender differences [5-7]. In a study conducted by Kirby et al. the amount of alcohol consumed per week correlated with the severity of psoriatic skin lesions [8]. The risk for incident psoriasis was found to be significantly high with the consumption of non-light beer (in an amount of $\geq 5$ drinks per week) but not with light-beer or other beverages, in a study of female nurses in USA [9]. McAleer et al. did not observe any significant relationship between excessive alcohol consumption and psoriasis severity [1]. In a similar way, disease severity did not differ significantly with alcohol consumption in the study performed by Serwin et al. Only the consumption of high grade alcoholic beverages did correlate with PASI value in male patients with a long history of psoriasis duration [10]. The aims of this study were a) to assess the alcohol consumption in the hospitalized psoriatic patients via CAGE questionnaire, their self- reports and the objective measurement of alcoholic markers in serum/ blood and b) to investigate the correlation between alcohol intake and psoriasis severity. 


\section{METHODS}

\section{Participants}

A total of 62 patients with psoriasis hospitalized at the Dermatology Clinic at "Mother Teresa" University Hospital in Tirana, Albania were the target population included in this cross-sectional study between November 2011 and November 2012. Patients with the presence of hepatic abnormalities and under the age 15 were excluded. The objectives of the study and confidentiality issues were discussed with the patients and their written consent was taken. The study protocol was approved by the Ethics Committee of "Mother Teresa" University Hospital.

\section{Data Gathering}

Data gathering was accomplished via face to face interviews performed by trained dermatology residents. Patients were asked about the basic sociodemographic data, psoriasis duration, family history of psoriasis, hospitalization frequency, alcohol consumption and smoking (pack per year). CAGE questionnaire was used to screen for chronic alcohol users, who were also asked to report the type and the amount of alcoholic beverage consumed per week (Table 1). The questions focusing on Cutting down, Annoyance by criticism, Guilty feeling and Eyeopeners, constituting the CAGE questionnaire, have proved useful in making a diagnosis of alcoholism [14] making this questionnaire a simple, sensitive and specific tool in screening for alcohol abuse or chronic alcohol use [15]. We did not test the reliability and validity of CAGE in our study, since this instrument has proven validity [16].

Patients consuming alcohol were classified as social drinkers (alcohol consumers on social occasions only), consumers of small amounts of alcohol, moderate amount of alcohol consumers and heavy drinkers. Subjects were also asked for the other types of alcohol used (whiskey, vodka, brandy), but since no other type of beverage was mentioned, we did not include it in our analysis. Beer and wine consumption were also initially divided into light/ non- light and red/ white, respectively; in this case also, non- light beer was the only mentioned beverage. Only one subject consumed white wine and this was included under wine subdivision.

\section{Measurements}

Disease severity was assessed via Psoriasis Area and Severity Index (PASI) scoring system, which consists of two steps: calculation of body surface area involved and evaluation of lesion severity according to the degree of erythema, induration and scaling, combining then all the calculations in a single score ranging from 0 (no disease) to 72 (all Body Surface Area (BSA) involved by the disease) [11]. According to PASI scores psoriasis severity among our subjects was classified as mild (PASI [2-12]), moderate PASI ((12$18])$ and severe (PASI > 18) [10].

According to psoriasis duration in years patients were categorized as the following: patients with psoriasis lasting less than 1 year, between 1 - 3 years and those with psoriasis duration of more than 3 years. Blood samples were taken within 24 hours of admission and the values of gamma glutamyltransferase (GGT), aspartate aminotransferase (AST), alanine aminotransferase (ALT) and erythrocyte mean corpuscular volume (MCV) were measured.

\section{Statistical Analysis}

Pearson coefficient was used for correlation analysis and regression coefficient was computed from the results of logistic regression analysis. Correlation coefficients between $0.1-0.3$ were considered low, between 0.31- 0.7 were considered moderate and those between 0.71- 1 were considered high. All tests of significance were two-tailed and a $P$-value of 0.05 or less was regarded as significant. Statistical analysis was conducted via statistical package SPSS 20.0.

\section{RESULTS}

\section{Participants}

The study included a total of 62 patients (29\% females, $71 \%$ males). The data on patients

Table 1: Alcoholic Beverages Consumed by Subjects in Type and Amount

\begin{tabular}{|c|c|c|c|}
\hline & $\begin{array}{c}\text { Small amount } \\
(\mathbf{m l} / \text { week })\end{array}$ & $\begin{array}{c}\text { Moderate amount } \\
(\mathbf{m l} / \text { week })\end{array}$ & $\begin{array}{c}\text { High amount } \\
(\mathbf{m l} / \text { week })\end{array}$ \\
\hline \hline Raki & $<50$ & $50-100$ & $>100$ \\
\hline Beer & $<500$ & $500-1000$ & $>1000$ \\
\hline Wine & $<200$ & $200-500$ & $>500$ \\
\hline
\end{tabular}


Table 2: Characteristics of Patients

\begin{tabular}{|c|c|c|c|}
\hline Characteristics of patients & Value & Standard Deviation & Range \\
\hline Total number of patients & 62 & - & - \\
\hline Male number & $44(71 \%)$ & - & - \\
\hline Female number & $18(29 \%)$ & - & - \\
\hline Average age in years & 44.95 & 13.10 & [15-64] \\
\hline Average age in years (males) & 45.25 & 13.06 & [15-63] \\
\hline Average age in years (females) & 44.22 & 13.57 & [15-64] \\
\hline Average disease duration & 13.19 & 11.47 & {$[1-40]$} \\
\hline Presence of psoriasis family history & $7(11.3 \%)$ & - & - \\
\hline Alcohol use & $27(43.5 \%)$ & - & - \\
\hline Smoking & $29(46,8 \%)$ & - & - \\
\hline Unemployment & $19(30.6 \%)$ & - & - \\
\hline
\end{tabular}

characteristics are as shown in Table 2. Based on the claimed data on occupation, the majority of our patients $(96.7 \%)$ did belong to the low socioeconomic class, that is they did a job that at most requires high school education. $12.9 \%$ of patients were social drinkers, 43.5 $\%$ did consume alcohol and the same percentage of patients did not consume alcohol at all. 24 out of 62 patients consuming alcohol (raki, beer and/ or wine) in high amounts were considered as heavy drinkers and thus constituting $39 \%$ of the total number of participants, $3 \%$ were moderate drinkers and $1.5 \%$ of participants consumed alcohol in small amounts.

\section{Measurements}

The mean PASI value was $26,03 \pm 15,93$ (range 1,8- 65,1). The mean PASI value was $17,11 \pm 11,07$ in females and 29,69 $\pm 16,26$ in males. The majority of patients had severe psoriasis (57\%) being followed by moderate psoriasis (24\%) and mild psoriasis (19\%) patients.

$19.4 \%$ of patients had a duration of psoriasis less than 3 years and $80.6 \%$ had psoriasis lasting more than 3 years.

A moderate but significant correlation was observed between GGT value with raki and beer consumption. The same moderate and positive correlation was seen between AST with beer and raki consumption. ALT did positively although moderately correlate with all the three types of alcoholic beverages, while MCV value did moderately correlate with raki consumption and strongly and positively correlate with beer consumption. A detailed description of significance levels and correlation coefficients is shown in Table 3.

Table 3: Correlations Between Alcoholic Biomarkers and the Amount of Alcohol Consumed

\begin{tabular}{|c|c|c|c|c|}
\hline \multirow{6}{*}{ 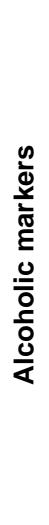 } & & \multicolumn{3}{|c|}{ Amount of alcohol consumed per week $(\mathrm{mL})$} \\
\hline & & Raki & Beer & Wine \\
\hline & GGT & $\begin{array}{l}r=0.45 \\
p<0.01^{*}\end{array}$ & $\begin{array}{l}r=0.36 \\
p=0.04\end{array}$ & $\begin{array}{c}r=0.05 \\
p=0.7\end{array}$ \\
\hline & AST & $\begin{array}{c}r=0.48 \\
p<0.01^{*}\end{array}$ & $\begin{array}{c}r=0.63 \\
p<0.01^{*}\end{array}$ & $\begin{array}{l}r=0.23 \\
p=0.07\end{array}$ \\
\hline & ALT & $\begin{array}{l}r=0.36 \\
p<0.01^{*}\end{array}$ & $\begin{array}{l}r=0.55 \\
p<0.01^{*}\end{array}$ & $\begin{array}{l}r=0.34 \\
p<0.01^{*}\end{array}$ \\
\hline & MCV & $\begin{array}{l}r=0.31 \\
p=0.01\end{array}$ & $\begin{array}{l}r=0.98 \\
p=0.04\end{array}$ & $\begin{array}{c}r=-0.44 \\
p=0.98\end{array}$ \\
\hline
\end{tabular}

$r=$ correlation coefficient

$p=p$ value.

${ }^{*}=$ highly significant $p$ value. 
When analyzing the amount of alcohol consumed in correlation to PASI score, the disease severity did not differ significantly with alcohol consumption ( $p>0.05$ ). No significant correlation was observed between PASI value with either raki or beer consumption. A significant and positive although moderate correlation was noted between PASI value in all individuals and wine consumption $(r=0.37, p<0.01)$ and PASI value in males with wine consumption ( $r=0.34, p<0.05)$. Logistic regression analysis between PASI score and raki consumption in male patients with psoriasis duration of more than 3 years resulted in statistical significance $(b=23.5$, Chi- Square value $=9.7, p<0.05)$. The same analysis has shown no association between psoriasis severity and beer/ wine consumption with the above mentioned category.

No significant association was observed between PASI score, alcohol consumption and employment status.

\section{DISCUSSION}

Our study has confirmed the literature results and the efficacy of clinical experience of traditional markers like gamma glutamyltransferase (GGT), aspartate aminotransferase (AST), alanine aminotransferase (ALT) and mean corpuscular volume of erythrocytes (MCV) in the identification of alcohol users [12, 13]. These alcoholic biomarkers have positively correlated with alcohol consumption, especially with raki consumption. One explanation can be the fact that raki consumption was more popular among our patients when compared to beer or wine consumption. This can also be related to the relatively affordable price of raki and the low socioeconomic status of our in- patients. Indeed, the correlations we found between alcohol consumption and the values of these markers were moderate in the majority of cases. We think that other factors might have contributed to these results, including age, gender, medications, herbal preparations, medical conditions like obesity, diabetes, hypertension, thyroid problems and smoking [4]. Similar results were observed by Poikolainen et al. in male psoriatic patients in Finland with moderate correlations between GGT and alcohol consumption ( $r=$ $0.35, p<0.01)$ and between AST and alcohol intake $(r=$ $0.3, p<0.01)[7]$.

Most of our patients had severe psoriasis, their disease was resistant to either topical or systemic treatment, the majority of them were males and they did consume alcohol significantly more than female psoriatic patients, a fact already confirmed in previous studies $[6,7]$. We think that multiple factors contribute to these outcomes. Apart from genetic predisposition, immune system response and other factors contributing to psoriasis severity, alcohol abuse commonly encountered in these patients together with low socio- economic status can be a burden to their disease severity.

As shown in this study, identification of alcohol consumers with CAGE questionnaire, followed by a self- reported amount of alcoholic beverages type and amount, combined with the measurement of affordable and readily accessible parameters like GGT, AST and ALT in serum and MCV in erythrocytes is a simple way of helping dermatologists dealing with alcohol issues in psoriatic patients.

Regarding the results of this study no correlation between psoriasis severity and alcohol consumption could be established. Indeed, a positive correlation was found between wine consumption and psoriasis severity $(r=0.37, p<0.01)$, however we did not regard this result as valid due to the small percentage of our patients consuming it, their low socioeconomic status and the possible consumption of wine made by traditional techniques.

On the other hand we did observe a significant association between raki consumption and psoriasis severity in male patients with a long duration of psoriasis $(b=23.5, p<0.05)$. Similar results were observed in the study by Serwin et al. except that the consumption of high grade alcoholic beverages as a group did correlate with psoriasis severity in male patients with long- lasting disease ( $R=0.41, p<0.05$ ), with no significant association found between beer or wine consumption with psoriasis severity $(-0.18<R<$ $0.13)$ [10]. On the other hand Kirby et al. found a significant association between physical severity of psoriasis and weekly alcohol consumption $(r=0.27, p<$ $0.05)[8]$.

We believe this finding makes our study unique since the specific role of raki in psoriasis severity has not been previously tested. Raki is a high grade alcoholic beverage that is traditionally and massively consumed among the Albanian people, men mostly. It contains about $45 \%$ alcohol and is consumed plain, not mixed with ice or water as Turkish people do. It is similar to ouzo consumed by the Greek or grappa consumed by Italians [17]. 
Still our study has several limitations. We could not include other markers of alcohol use as carbohydratedeficient transferrin (CDT) shown to be superior to other markers, due to its being not available as a test in our laboratories. We could not test the correlation of alcoholic markers with the amount of alcohol consumed as far as it concerns the gender differences, since our female patients consuming alcohol constituted a small part of the social drinker category only. Other beverages like non- light beer or those containing high grade alcohol were not the favorite of our patients, probably due to their low socioeconomic status and male predominance. Obviously, further studies and a more heterogenous group of psoriasis patients (in terms of socioeconomic status) are needed to assess the increased severity of the disease in patients consuming alcohol.

\section{ACKNOWLEDGEMENTS}

The authors would like to thank patients for their participation in the study and the authors of the articles that have been influential in this paper: Psoriasis and unreported excessive alcohol intake--a simple screening approach by Pietrzak A et al. and Selenium status in psoriasis and its relationship with alcohol consumption by Serwin $\mathrm{AB}$ et al.

\section{APPENDIX}

PASI = psoriasis area and severity index

GGT = gamma glutamyltransferase

AST = aspartate aminotransferase

ALT = alanine aminotransferase

$\mathrm{MCV}=$ mean corpuscular volume

CDT = carbohydrate- deficient transferrin

$\mathrm{BSA}=$ body surface area

$r=$ correlation coefficient

b $=$ regression coefficient

$\mathrm{p}=\mathrm{p}$ value

$\mathrm{R}=$ Spearman's rank coefficient

\section{CONFLICTS OF INTEREST}

None.

\section{FUNDING SOURCES}

None.

\section{REFERENCES}

[1] McAleer MA, Mason DL, Cunningham S, et al. Alcohol misuse in patients with psoriasis: identification and relationship to disease severity and psychological distress. Br J Dermatol 2011; 164(6): 1256-61. http://dx.doi.org/10.1111/j.1365-2133.2011.10345.x

[2] Del Boca FK, Darkes J. The validity of self-reports of alcohol consumption: state of the science and challenges for research. Addiction 2003; (98 Suppl 2): 1-12. http://dx.doi.org/10.1046/j.1359-6357.2003.00586.x

[3] Alte D, Luedemann J, Rose HJ, John U. Laboratory markers carbohydrate-deficient transferrin, gammaglutamyltransferase, and mean corpuscular volume are not useful as screening tools for high-risk drinking in the general population: results from the Study of Health in Pomerania (SHIP). Alcohol Clin Exp Res 2004; 28(6): 931-40. http://dx.doi.org/10.1097/01.ALC.0000128383.34605.16

[4] Pietrzak A, Jastrzębska I, Chodorowska G, et al. Psoriasis and unreported excessive alcohol intake--a simple screening approach. J Eur Acad Dermatol Venereol 2011; 25(11): 1261-8. http://dx.doi.org/10.1111/j.1468-3083.2011.04101.x

[5] Wolf R, Wolf D, Ruocco V. Alcohol intake and psoriasis. Clin Dermatol 1999; 17: 423-30. http://dx.doi.org/10.1016/S0738-081X(99)00028-0

[6] Gupta MA, Schork NJ, Gupta AK, Ellis CN. Alcohol intake and treatment responsiveness of psoriasis: a prospective study. J Am Acad Dermatol 1993; 28(5 Pt 1): 730-2. http://dx.doi.org/10.1016/0190-9622(93)70101-X

[7] Poikolainen K, Reunala T, Karvonen J, Lauharanta J, Kärkkäinen P. Alcohol intake: a risk factor for psoriasis in young and middle aged men? BMJ 1990; 300(6727): 780-83. http://dx.doi.org/10.1136/bmj.300.6727.780

[8] Kirby B, Richards HL, Mason DL, Fortune DG, Main CJ, Griffiths CE. Alcohol consumption and psychological distress in patients with psoriasis. $\mathrm{Br} \mathrm{J}$ Dermatol 2008; 158(1): 13840. Epub 2007 Nov 10. Reference: Available from: http://www.ncbi.nlm.nih.gov/pubmed/17999698

[9] Qureshi AA, Dominguez PL, Choi HK, Han J, Curhan G. Alcohol Intake and Risk of Incident Psoriasis in US Women, A Prospective Study. Arch Dermatol 2010; 146(12): 1364-69. http://dx.doi:10.1001/archdermatol.2010.204

[10] Serwin AB, Wasowicz W, Gromadzinska J, Chodynicka Z. Selenium status in psoriasis and its relationship with alcohol consumption. Biol Trace Elem Res 2002; 89: 127-40. http://dx.doi.org/10.1385/BTER:89:2:127

[11] PASI training [homepage on the Internet] [2009] Available from: http://www.pasitraining.com/about.html

[12] Sharpe PC. Biochemical detection and monitoring of alcohol abuse and abstinence. Rev Article Ann Clin Biochem 2001; 38: $652-64$.

[13] Conigrave KM, Davies P, Haber P, Whitfield JB. Traditional markers of excessive alcohol use. Addiction 2003; 98(Suppl 2): $31-43$. http://dx.doi.org/10.1046/i.1359-6357.2003.00581.x

[14] Ewing JA. Detecting alcoholism: the CAGE questionnaire. JAMA 1984; 252(14): 1905-907 http://dx.doi.org/10.1001/jama.1984.03350140051025

[15] Bush B, Shaw S, Cleary P, Delbanco TL, Aronson MD. Screening for alcohol abuse using the CAGE questionnaire. Am J Med 1987; 82(2): 231-5.

http://dx.doi.org/10.1016/0002-9343(87)90061-1 
[16] Dhalla S, Kopec JA. The CAGE questionnaire for alcohol misuse: a review of reliability and validity studies. Clin Invest Med 2007; 30(1): 33-41.
[17] Facts and Details [homepage on the Internet] [Last updated March 2011] Available from: http://factsanddetails.com/world. php? itemid $=1554 \&$ subcatid $=347$ 